Revue scientifique sur la conception et l'aménagement de l'espace

\title{
La forêt comme inspiration et image-outil de coconception
}

Le pavillon de la Suède à l'Expo 2020 Dubaï

The Forest as an Inspiration and a Co-Designing Image-Tool - Sweden's pavilion at Expo 2020 Dubai

\section{Marina Khémis}

\section{(2) OpenEdition}

Journals

Édition électronique

URL : http://journals.openedition.org/paysage/8446

DOI : 10.4000/paysage. 8446

ISSN : 1969-6124

\section{Éditeur :}

École nationale supérieure du paysage de Versailles-Marseille, Institut national des sciences appliquées Centre Val de Loire - École de la nature et du paysage, École nationale supérieure d'architecture et de paysage de Bordeaux, École nationale supérieure d'architecture et de paysage de Lille, Agrocampus Angers

Référence électronique

Marina Khémis, "La forêt comme inspiration et image-outil de coconception », Projets de paysage [En ligne], 22 | 2020, mis en ligne le 21 juillet 2020, consulté le 25 juillet 2020. URL : http:// journals.openedition.org/paysage/8446; DOI : https://doi.org/10.4000/paysage.8446

Ce document a été généré automatiquement le 25 juillet 2020.

Projets de paysage 


\title{
La forêt comme inspiration et image-outil de coconception
}

\author{
Le pavillon de la Suède à l'Expo 2020 Dubaï
}

The Forest as an Inspiration and a Co-Designing Image-Tool - Sweden's pavilion

at Expo 2020 Dubai

Marina Khémis

\section{« The Forest » : une forêt architecturale}

1 «The Forest » est le projet lauréat pour le pavillon de la Suède à l'Exposition universelle de 2020 à Dubaï ${ }^{1}$, conçu en équipe par Alessandro Ripellino Arkitekter, le Studio Adrien Gardère et Luigi Pardo Architetti. Ce projet s'appuie sur l'image et la symbolique de la forêt aussi bien d'un point de vue architectural, programmatique, curatorial, scénographique que paysager. Au-delà d'une simple dimension esthétique ou visuelle, la forêt a été - en tant que telle - un véritable outil de coconception, guidant le projet dans chacun de ses aspects, étapes et échelles. De l'intuition de départ et du déploiement de sa portée symbolique, culturelle, scientifique, spatiale et sensible, aux réponses aux diverses contraintes à la fois fonctionnelles et narratives du programme, la forêt a systématiquement guidé chacune des décisions et orientations prises au fil du projet, selon un processus itératif et non linéaire, croisant les apports complémentaires de chacun des membres de l'équipe.

2 Nous chercherons à montrer en quoi la mise en place d'une démarche de collaboration en amont du projet, fondée sur le partage d'une idée commune, permet de générer une expérience totale pour le visiteur, où architecture, scénographie, contenus et récit forment un tout. 
Figure 1. Vue perspective du projet depuis l'allée principale de l'Expo

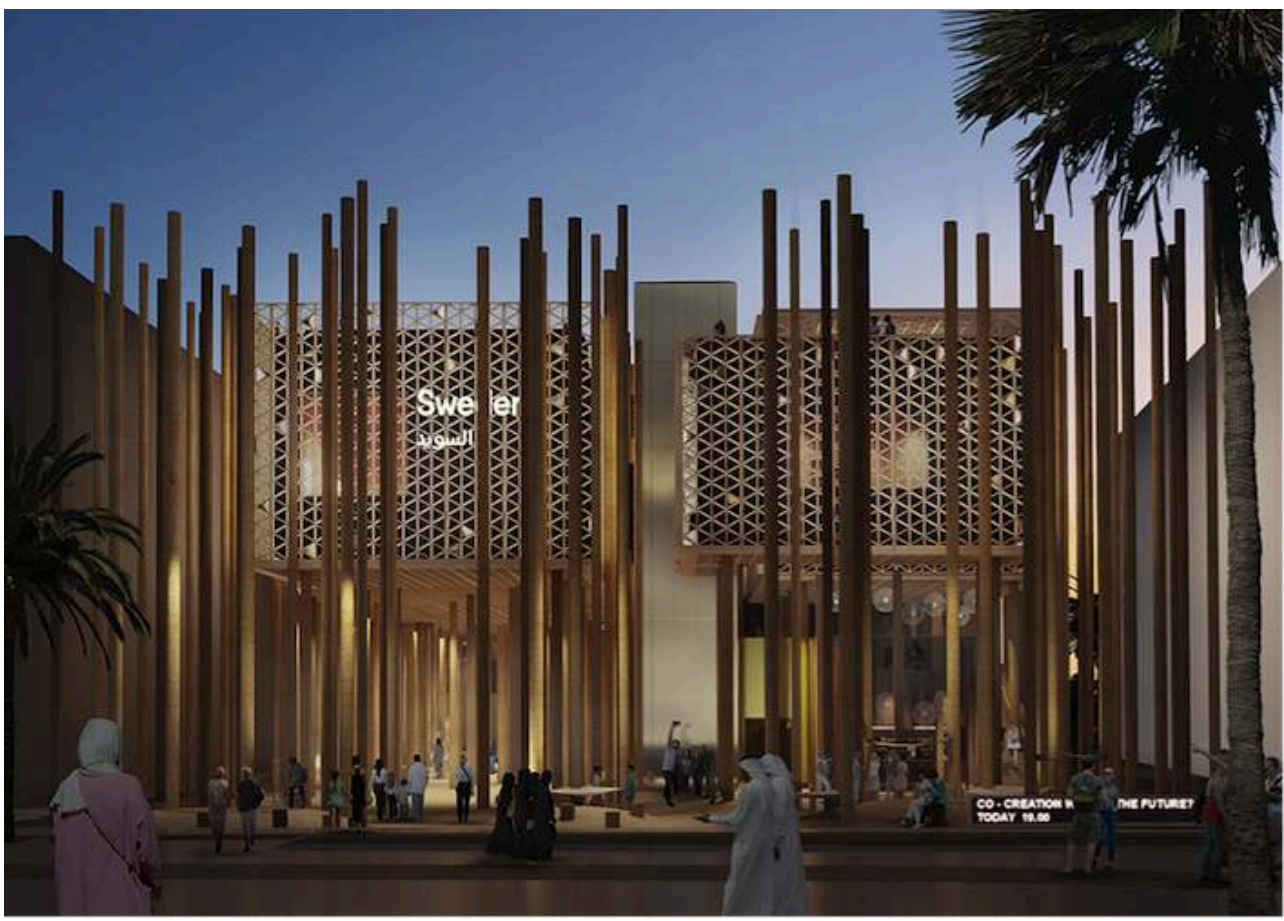

Sources : Alessandro Ripellino Arkitekter + Studio Adrien Gardère + Luigi Pardo Architetti.

\section{La forêt comme intuition d'équipe}

3 La volonté d'une approche holistique du projet capable de produire « un concept clé et une approche claire, aussi bien d'un point de vue constructif qu'expositif » faisait partie des attendus de la maîtrise d'ouvrage dès la phase de candidature. La demande du programme exigeait donc la conception aussi bien de l'architecture du pavillon que de son exposition et de sa scénographie.

Pour répondre à cette attente, notre équipe s'est constituée dès l'origine autour de compétences multiples et complémentaires: celles de l'agence d'architecture Alessandro Ripellino Arkitekter, basée à Stockholm ; celles des architectes italiens Luigi Pardo Architetti, basés à Rome, spécialisés dans la recherche sur l'architecture durable ; et celles du Studio Adrien Gardère, basé à Paris ${ }^{2}$ et spécialisé en muséographie, design et scénographie.

5 Lors de la phase de concours, l'analyse du programme menée par notre équipe a permis de repérer quatre axes clés :

1. d'un point de vue constructif, compte tenu de l'importance et du soutien de l'industrie suédoise de la filière bois, la nécessité de construire le pavillon en bois ;

2. en termes fonctionnels, la nécessité de concevoir des espaces d'exposition et de conférences, aussi bien intérieurs qu'extérieurs, ouverts au public, mais aussi - de manière plus privée aux invités et professionnels ;

3. en matière de récit et de contenus, l'accent mis sur le thème de la cocréation et de la nature collective de la créativité et de l'innovation en Suède et plus largement du caractère inclusif de sa société. En bref, offrir une image forte et positive de la Suède. L'exposition doit présenter les 4 grands secteurs de l'innovation suédoise ; 
4. enfin les attentes très importantes du programme en matière de développement et de construction durables; de prise en compte des enjeux climatiques à l'échelle mondiale et à celle du site de l'exposition : les Émirats arabes unis.

6 La forêt émerge dès les premiers échanges au sein de l'équipe (figure 2), non seulement en tant qu'image, pour sa capacité à produire un signe architectural fort, son caractère emblématique suédois, mais aussi en tant que concept et modèle vivant de coexistence, de symbiose naturelle et de cycles de cocréation, en réponse aux enjeux du programme.

Figure 2. Croquis réalisés par l'équipe lors du lancement de la phase de concours
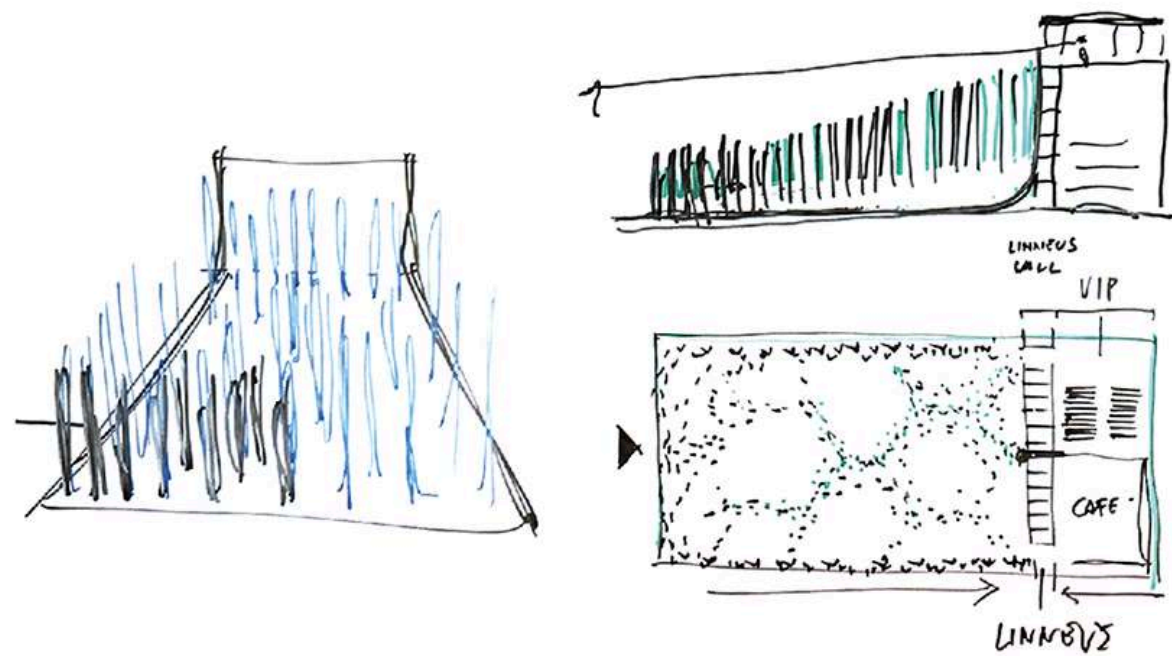

Ils représentent l'intention architecturale de la forêt, en vue surplombante, élévation et plan.

Sources : Alessandro Ripellino Arkitekter + Studio Adrien Gardère + Luigi Pardo Architetti.

7 Partant de cette intuition, une première stratégie de collaboration se met en place : chacun des membres du groupement se concentre sur des aspects parallèles et complémentaires de la "forêt » afin d'étoffer et de donner corps à notre intention de départ:

- Les architectes Alessandro Ripellino Arkitekter développent dans un premier temps la répartition des fonctions et leur interconnexion, avec des premières hypothèses de mise en forme du programme, tout en cherchant à rester fidèles à la notion de forêt.

- Les architectes Luigi Pardo Architetti approfondissent les questions climatiques de la forêt et les opportunités qu'elle offre en matière de gestion et d'apport de lumière et de chaleur dans le contexte des Émirats arabes unis.

- Enfin, le Studio Adrien Gardère se concentre dans un premier temps sur la construction du récit et du propos afin de tirer pleinement parti de l'idée de forêt, non seulement en tant qu'espace typique suédois, mais aussi dans sa dimension symbolique, politique, voire spirituelle, et enfin en tant qu'espace et lieu d'exposition, de visite et d'expériences.

L'image de forêt et son fonctionnement en tant qu'écosystème ont inspiré toute l'équipe et l'ont amenée à dépasser la dissociation fréquemment faite dans la conception des lieux culturels entre enveloppe architecturale, contenus, récit et scénographie d'exposition. 
9 Ici il n'y a pas, d'une part, une boîte architecturale et, d'autre part, un contenu qui l'occupe : les expertises de chacun des membres de l'équipe sont mobilisées sur chaque composante du bâtiment, associant des réflexions transversales et complémentaires selon des processus croisés et non linéaires.

\section{De l'image à l'espace : déployer la portée de la forêt en équipe}

10 Afin de passer de l'intuition à la construction d'une narration, puis à son déploiement dans l'espace, nous avons mené au Studio Adrien Gardère une exploration de la notion de "forêt ", alimentée à la fois par nos expériences et nos imaginaires propres, mais aussi par la consultation de textes littéraires, scientifiques et philosophiques, d'émissions, de vidéos, ou de travaux d'artistes ${ }^{3}$, afin d'approfondir notre compréhension de l'écosystème de la forêt, de son réseau de communication, de ses habitants - réels ou imaginaires -, de toute la symbolique et l'imaginaire dont elle est porteuse. Nos recherches, complétées par des échanges notamment avec l'équipe d'architectes suédois et des chercheurs suédois, nous ont aussi permis d'appréhender les spécificités de la forêt suédoise, ses caractéristiques physiques mais aussi culturelles et mythologiques.

11 La forêt est un lieu de retraite et d'inspiration, de mystères et de mythes, elle est, comme la décrit Robert Harrison (1994), un lieu à la fois inquiétant et enchanteur, de perdition et de refuge, où nature et culture s'opposent et se rencontrent, où dans la densité des bois se dessine l'espace lumineux des clairières où l'homme s'implante pour $\mathrm{y}$ construire ses cabanes. La forêt est aussi un écosystème où la nature innove en permanence, un réseau de communication et d'individus interdépendants et fonctionnant comme un tout. Comme le révèle notamment Peter Wohlleben (2017), la forêt, par son univers souterrain de racines et de champignons interconnectés, mais aussi par l'ensemble des messages et signaux transmis par l'air, constitue un véritable système de collaboration et d'entraide. La forêt apparaît dès lors comme le symbole parfait de la thématique de la cocréativité et de l'innovation choisie par la Suède pour son pavillon.

12 À ces recherches s'ajoutent celles menées sur le «double » de la forêt: le désert des Émirats arabes unis, où se tiendra l'exposition universelle. La mise en regard entre la nature et la culture de la forêt et celles du désert est en effet une des inspirations du projet.

13 La forêt et le désert sont tous deux des lieux de création, de ressourcement et de retraite spirituelle, mais aussi d'inscription au monde : l'anachorète d'un côté, l'ermite ${ }^{4}$ de l'autre. Ce sont aussi des espaces " habités » d'esprits et de légendes: elfes, lutins, trolls, gnomes, dans l'une ; djinns, efrits, sylphes ${ }^{5}$ et mirages dans l'autre.

14 Enfin, dans la perspective des enjeux de développement durable du projet, la forêt et le désert sont par excellence des lieux où l'homme apprend à vivre en harmonie avec la nature et où le bon usage des ressources naturelles est de règle pour survivre. Ces parallèles nous permettent ainsi " d'enraciner » notre concept de forêt dans le désert et d'inscrire le projet dans un dialogue entre la culture suédoise et celle de nos interlocuteurs et hôtes émiratis. 
L'ensemble de ces réflexions donneront naissance au texte d'introduction des planches de rendu du concours (figure 3), maintenant la cohérence de notre concept tout au long du projet, aussi bien pour l'architecture et la scénographie que pour le déploiement d'une narration et la construction des contenus de l'exposition.

Figure 3. Texte manifeste « The Forest As »

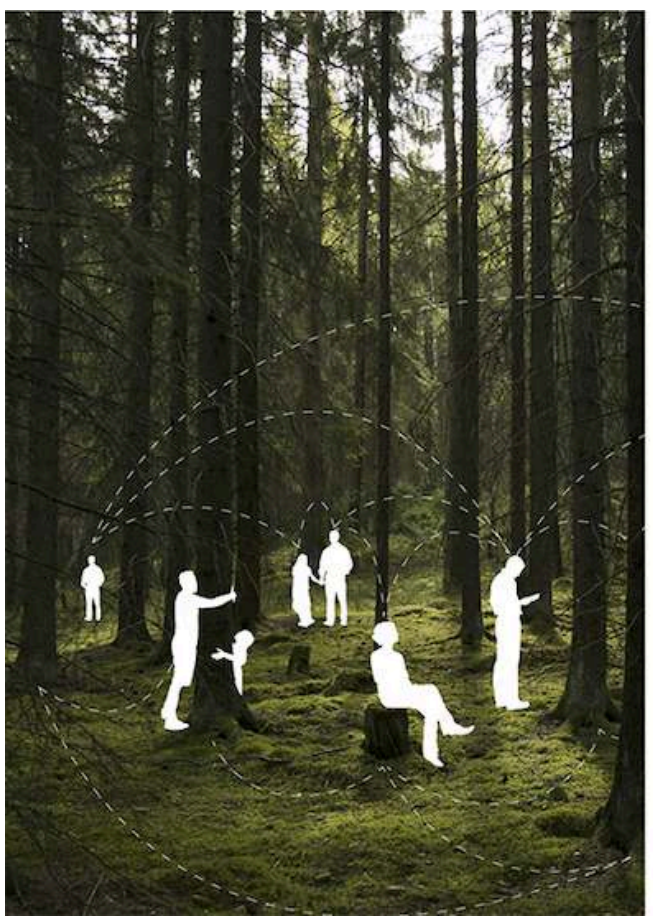

The Forest as ...

an emblematic image of Swede

a sanctuary and growing ecosystem

a universal place of retreat and creative withdrawal

a place of remoteness and solitude, echoing the desert of UAE

a place of unexpected encounters

a cradle for myths, legends and tales

a place of mystery, adventure and discovery

a realm of imagination, revelation and transformation

a network of creative grounds

a strong symbol of interconnection and mutual resources

a metaphor of connected individuals functioning as a whole

an ecosystem overcoming constraints and obstacles together

a natural way of ovoking Swoden to the world

Ce texte a été rédigé par le Studio Adrien Gardère lors de la phase de concours.

Sources : Alessandro Ripellino Arkitekter + Studio Adrien Gardère + Luigi Pardo Architetti.

16 Très rapidement, à la construction du récit par des mots s'ajoute sa traduction en un vocabulaire spatial et sensoriel, fondé sur un ensemble de dessins, de schémas (figures 4 et 5), d'images de références et d'installations artistiques.

17 En identifiant le potentiel architectural et scénographique de chacune des spécificités de la forêt, nous cherchons à définir l'environnement et les expériences que le visiteur retrouvera dans le pavillon.

Chacune des différentes typologies et caractéristiques de la forêt est étudiée : la densité des arbres, les clairières, l'orée du bois et ses marges diffuses, les souches d'arbres coupés, la topographie des sols et les formes qui en émergent, l'architecture souterraine des racines et les signaux invisibles; mais aussi les façons de s'orienter et de se repérer dans la forêt avec notamment le marquage des arbres pour indiquer un chemin de randonnée. À ce vocabulaire s'ajoutent l'atmosphère et les sensations propres à la forêt : la manière dont la lumière traverse la canopée, le son du vent et des animaux, la souplesse du sol couvert de feuilles. 
Figure 4. Dessins de recherche

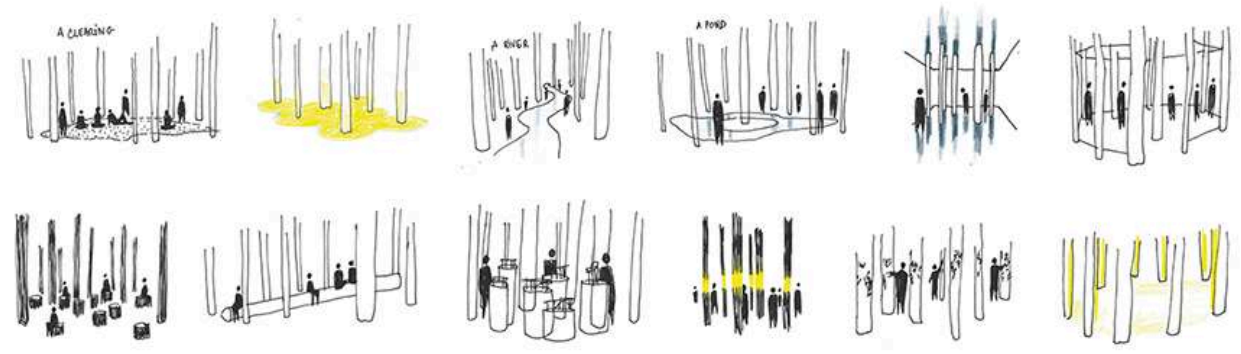

Réalisés en phase de concours pour explorer le vocabulaire spatial de la forêt : clairières, souches, marquages sur les arbres, etc.

Sources : Marina Khémis pour le Studio Adrien Gardère.

Figure 5. Représentations schématiques du vocabulaire spatial de la forêt issues du rendu de la phase de concours

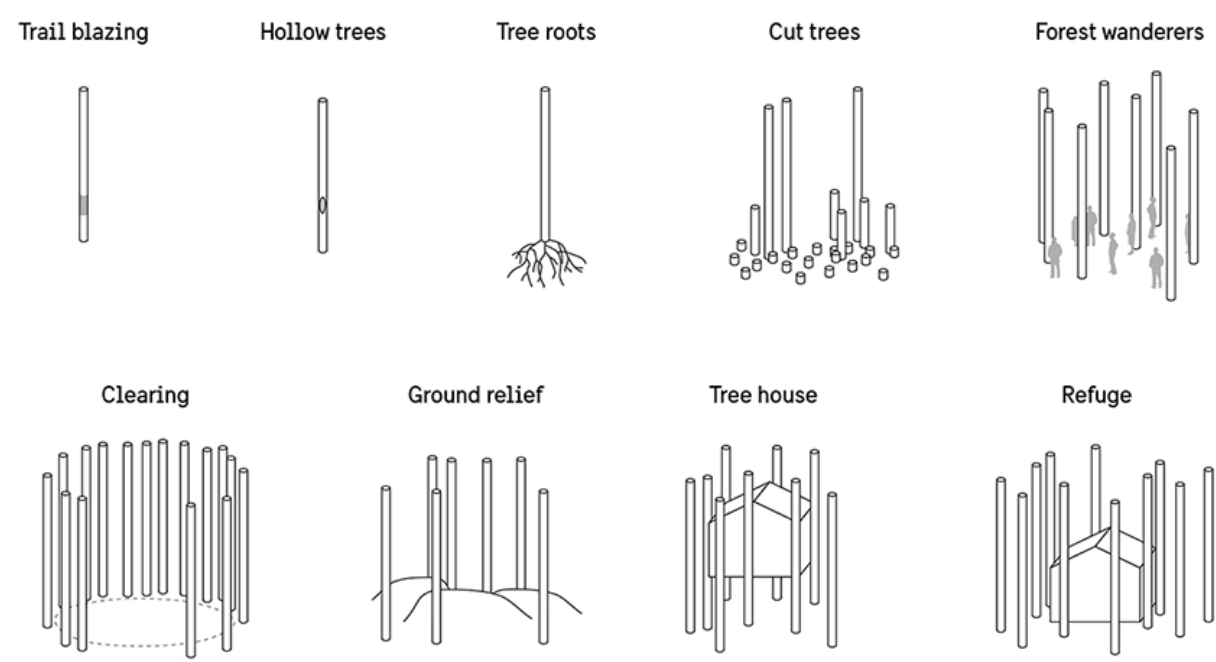

Source : Studio Adrien Gardère.

19 Ce croisement entre narration et mise en espace constitue le fondement du projet, il structure l'utilisation de l'image de la forêt développée en équipe, entre l'affirmation d'un symbole, d'une matérialité, de sensations, et la réponse aux demandes fonctionnelles et narratives du programme. Il s'agit, comme pour tout projet de scénographie, de parvenir à s'approprier et à dépasser la réalité programmatique pour construire un imaginaire et le déployer dans l'espace que les visiteurs s'approprieront à leur tour, construisant leur propre récit au fil de leurs déambulations.

Cela revient, comme le décrit Arnaud Sompairac (2016), à agencer un récit dans l'espace, créant un espace fictionnel conçu pour et par le déplacement du visiteur, et jouant des intervalles et interrelations entre les items exposés, les visiteurs et le contexte ou "paysage intérieur " dans lequel cela se déploie. L'architecture apparaît ainsi comme un élément à part entière du récit. 


\section{Construire une forêt architecturale : la rencontre des enjeux narratifs et programmatiques}

21 Tout en prenant racine dans la richesse et la diversité de ces premières réflexions, la mise en forme du projet en équipe cherche tout d'abord à déterminer, sous la forme la plus minimale possible, ce qui d'un point de vue architectural fait forêt. La réflexion en plan et en élévation permet notamment d'aborder cette question.

L'un des aspects essentiels explorés par le projet est cette notion de marge, de seuil, de ce qui délimite le pavillon, dans sa forme mais aussi dans sa perception, lorsque l'on cherche à représenter une forêt. Cette question est d'autant plus essentielle qu'elle fait directement référence au concept suédois - ou plus largement nordique - d' allemansrätten, correspondant au droit de déambuler librement dans la nature, principalement en forêt puisque $70 \%$ de la surface du pays en est recouverte.

Pour créer cette forêt architecturale, nous ne sommes donc pas partis d'une enveloppe ou d'une façade, mais de la concentration, de l'accumulation de la verticalité des troncs, afin de produire la matérialité enveloppante et diffuse, progressivement sculptée au fil du projet, par dilatation, retrait, ajout, densification, pour générer tout à la fois l'architecture du pavillon, le parcours de l'exposition et accueillir les fonctions du programme.

La question du positionnement des espaces privés de conférences et de leur rapport aux espaces publics d'exposition a été essentielle dans le dessin de notre projet architectural. Après avoir exploré différentes hypothèses, c'est la solution du positionnement de ces espaces privés en hauteur, au-dessus de la forêt, que nous choisissons d'adopter (figure 6). Ici, comme pour chaque aspect du projet, les enjeux du programme et les apports de chacune des expertises de l'équipe sont croisés, dans une logique de coconception, maintenant à chaque étape la ligne narrative du projet et l'image de la forêt.

Figure 6. Schéma d'analyse de l'évolution du projet et des accès publics et privés en élévation
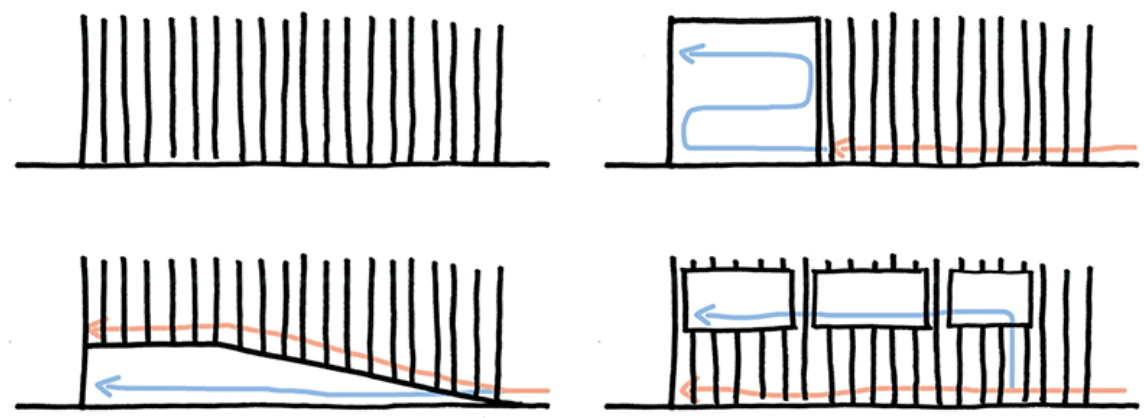

De la simple accumulation des troncs, aux options de positionnement des espaces de conférences (au fond, en dessous, en haut).

Source : Marina Khémis.

Le positionnement des espaces de conférences en partie haute permet à la fois :

- de répondre aux contraintes du climat et de l'ensoleillement, en offrant des espaces ombragés et plus frais en partie basse ; 
- de séparer les fonctions publiques et privées ;

- de créer une exposition accessible sans aucune discontinuité, de plain-pied avec le niveau de l'allée centrale du site.

Au-delà d'une réponse pragmatique, cette approche renforce l'imaginaire de la forêt, en donnant aux espaces privés de conférences le statut de « cabanes dans les arbres ».

En effet, la mise en forme architecturale de cet étage est conçue comme une série de boîtes portées par les troncs et connectées par des passerelles couvertes. Les écarts conservés entre les cabanes et leur matérialité permettent d'offrir des vues sur le ciel tout en filtrant la lumière du soleil. La très généreuse hauteur sous plafond, huit à neuf mètres au niveau de l'espace d'exposition, permet de maintenir la perspective filante des arbres. Dans la logique des marges diffuses, les espaces de repos et de récréation sont pleinement intégrés à la forêt. Situés sur le parvis du pavillon et dans la continuité de l'espace d'exposition, ils en constituent la lisière tout en symbolisant la culture suédoise d'invitation et d'ouverture (figure 7).

Figure 7. Dessins du projet réalisés en phase de concours
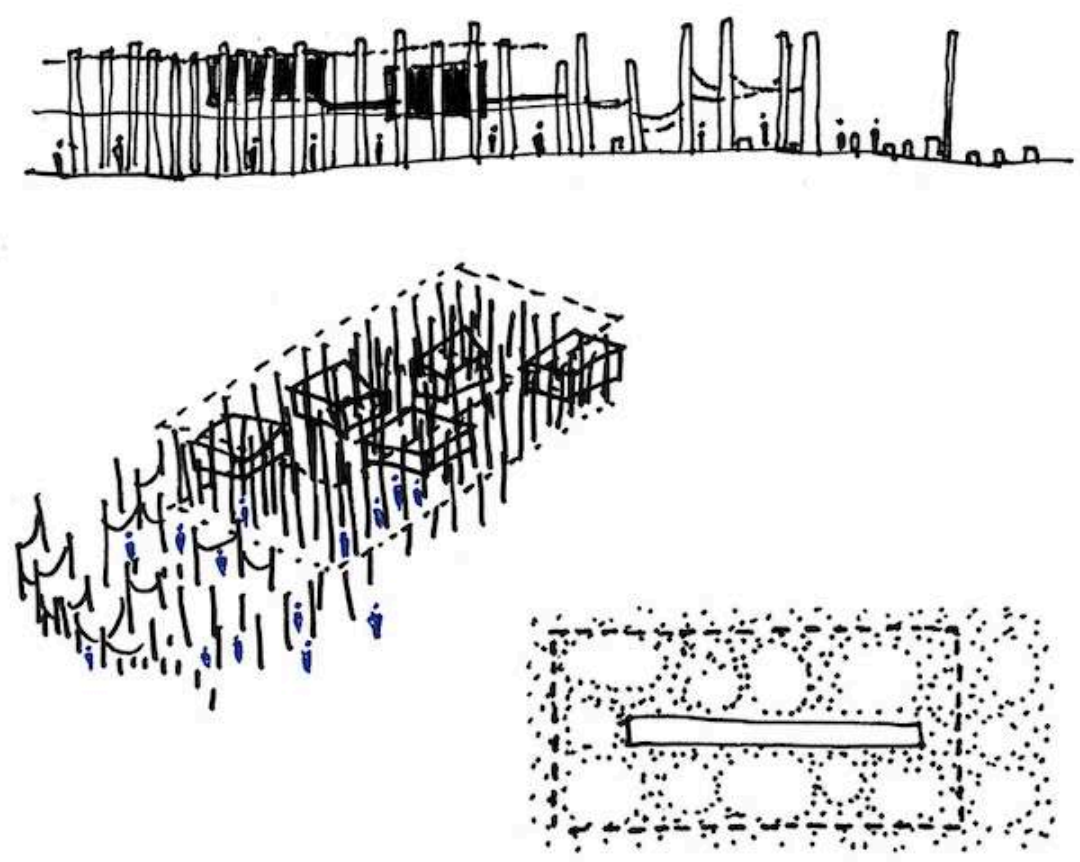

Ils représentent les cabanes dans les arbres et le déploiement des jeux sur le parvis, ainsi que le travail de clairières autour du mur central.

Sources : Marina Khémis pour le Studio Adrien Gardère/Alessandro Ripellino Arkitekter + Studio Adrien Gardère + Luigi Pardo Architetti.

$\mathrm{Au}$ rez-de-chaussée, le plan libre du pavillon destiné à accueillir l'exposition se compose par un jeu de densité: l'accumulation des troncs d'arbres, déployés sur l'ensemble de la surface du terrain, définit une série de clairières qui accueillent les différentes thématiques de l'exposition. Afin de clarifier les circulations entre ces clairières et d'éviter un parcours de visite trop libre, le Studio Adrien Gardère propose de créer au centre du pavillon une paroi centrale, permettant de former une boucle de 
visite claire. Les architectes Alessandro Ripellino s'emparent alors de ce nouvel élément architectural pour en faire un véritable mur habité, accueillant l'ensemble des circulations verticales. Ce mur permet de générer un point d'accès dédié aux invités des espaces de conférences, clairement dissocié de l'accès principal aux espaces d'exposition (figure 8). Puis la matérialité même de cette épine dorsale du pavillon devient un dispositif scénographique à part entière; recouverte d'un revêtement réfléchissant, cette paroi multiplie et prolonge la forêt, créant une sensation de profondeur en tout point de l'espace et renforçant l'expérience offerte au visiteur.

Figure 8. Schéma d'analyse de l'évolution du projet en plan ainsi que des circulations du public
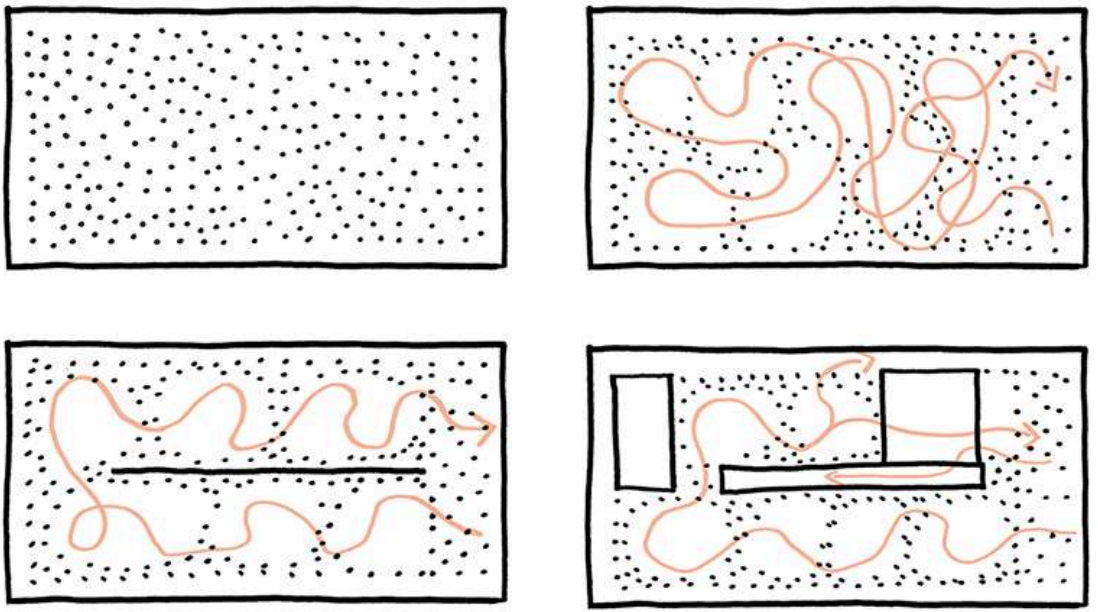

De l'accumulation des troncs sur le terrain à l'ouverture de clairières, jusqu'à la structuration du parcours par le mur central.

Source : Marina Khémis.

\section{Les formes, matières et récits d'une forêt fabriquée}

Au-delà des grands principes structurant l'architecture et les volumes du pavillon, l'image de la forêt se prolonge aussi dans le détail du projet - d'abord en phase de concours puis en échange direct avec la maîtrise d'ouvrage - guidant les choix de matérialité, de vocabulaire formel, de typologies de supports d'exposition, mais aussi les contenus mêmes de l'exposition et la mise en place d'un récit cohérent dans le parcours de visite. 
Figure 9. Vue perspective du pavillon et de la paroi réfléchissante depuis l'exposition

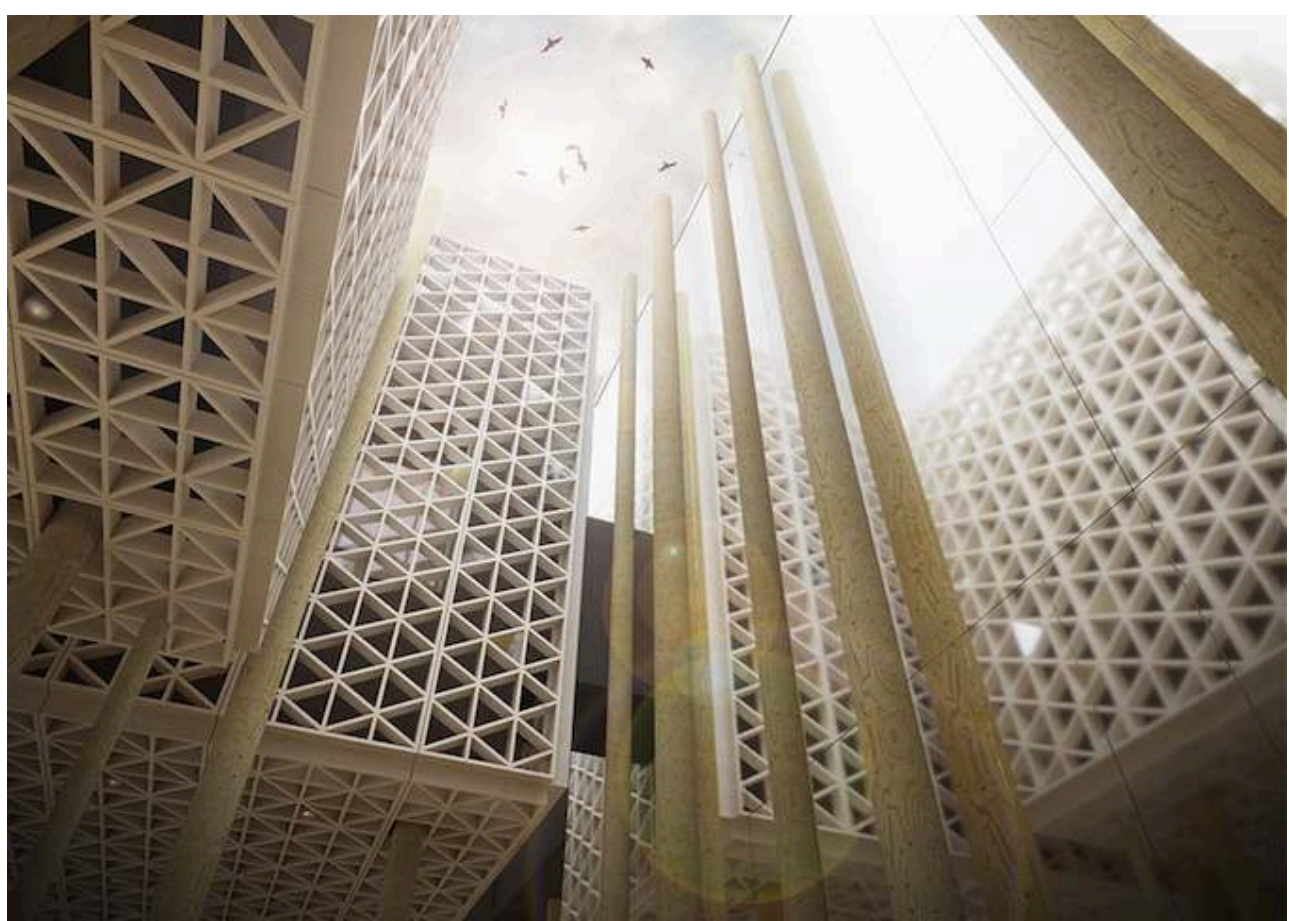

Sources : Alessandro Ripellino Arkitekter + Studio Adrien Gardère + Luigi Pardo Architetti.

La matérialité de l'architecture du pavillon correspond à l'association de plusieurs éléments issus du matériau bois. Un ensemble d'environ trois cents troncs d'arbres bruts sans écorce, de 8 à 18 mètres, forme le corps de la forêt. Les « cabanes ", perchées dans les arbres et reposant sur des troncs structurels, sont composées de panneaux de bois ajourés, placés à une légère distance de la structure des boîtes, et évoquant les motifs de moucharabieh ${ }^{6}$, en dialogue avec l'architecture locale (figure 9). Enfin, le sol du pavillon crée un léger jeu de topographie (figure 10), accompagnant la trame d'implantation des troncs et les flux des visiteurs et créant des zones de jeux sur le parvis. Le sol se compose d'un matériau à base de liège, dont la souplesse évoque la consistance du sol de forêt.

31 À ces différents composants du pavillon issus de matériaux naturels, sont associées des matérialités s'inscrivant davantage dans une logique d'effacement. Il y a, d'une part, le revêtement réfléchissant permettant d'effacer l'élément central du pavillon au profit d'une prolongation de l'accumulation des troncs et, d'autre part, les cloisons de verre délimitant l'espace du café et de la boutique au rez-de-chaussée. 
Figure 10. Deux plans du projet représentant la répartition des installations et le traitement de la topographie du sol
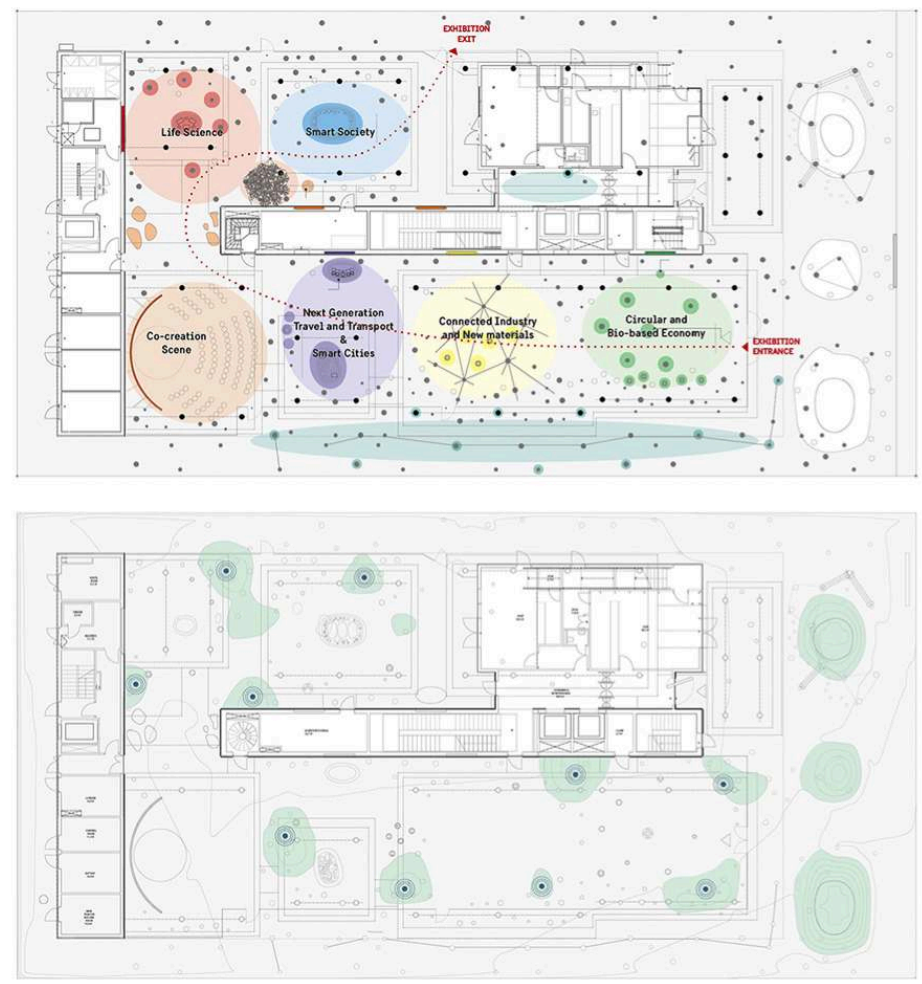

Sources : Alessandro Ripellino Arkitekter + Studio Adrien Gardère + Luigi Pardo Architetti.

$\mathrm{Au}$ sein de l'exposition, chacun des dispositifs de présentation et de médiation est subtilement intégré à la forêt. Les éléments de médiation multimédia s'intègrent à des supports en relief, pensés comme des émergences prolongeant la matérialité du sol. Les supports d'exposition et zones d'interactions ponctuelles reprennent la matérialité et la forme des troncs. C'est aussi dans cette même logique qu'est déployée sur le parvis et à travers le parcours de l'exposition une série de troncs bas, comme des souches d'arbres, permettant d'offrir des points d'assise (figure 11). Les troncs hauts deviennent aussi dans l'ensemble de l'exposition des supports de signalétique, à l'image des marquages en forêt indiquant les pistes à suivre. Ces troncs sont aussi ponctuellement le support d'animations lumière intégrées, jouant sur des interactions plus intuitives et sensibles avec les visiteurs. Le mur central réfléchissant sert de support à l'intégration discrète de contenus digitaux, avec des écrans dont la surface se fond dans la matérialité du mur. 
Figure 11. Vue perspective de l'exposition réalisée en phase de concours

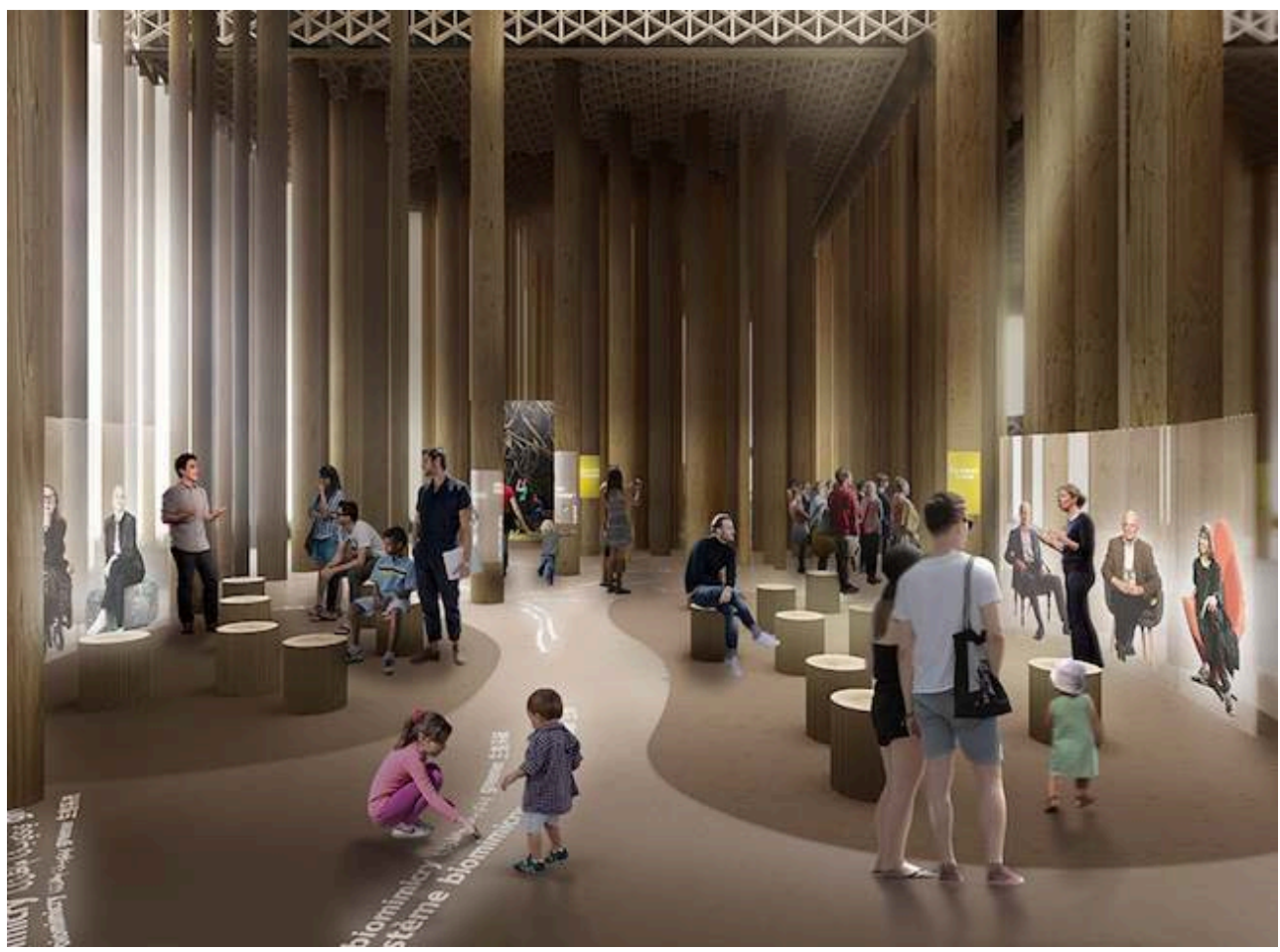

Sources : Alessandro Ripellino Arkitekter + Studio Adrien Gardère + Luigi Pardo Architetti.

33 C'est aussi en filant la symbolique de forêt que la cohérence des contenus de l'exposition est structurée. Le programme en phase de concours demeure en effet très ouvert quant au contenu de l'exposition, la demande est de communiquer sur les quatre grands secteurs de l'innovation suédoise: l'économie circulaire, l'industrie connectée et les nouveaux matériaux, les moyens de transport et la ville intelligente, les sciences du vivant et la santé.

La direction artistique de l'exposition et la structuration générale des contenus sont confiées au Studio Adrien Gardère, assurant ainsi une cohérence d'ensemble entre architecture, scénographie et contenus. À la suite du concours, c'est donc un autre niveau de coconception qui s'amorce, associant le Studio Adrien Gardère à la maitrise d'ouvrage ainsi qu'à l'ensemble des partenaires et des entreprises sponsors pour penser le récit déployé dans l'espace. La forêt devient alors un moyen d'accompagner la compréhension des différentes thématiques de l'exposition (figure 12). En tant qu'écosystème, elle représente le modèle circulaire par excellence, permettant par exemple de présenter les principes de bioéconomie mis en place par l'industrie forestière et permettant le stockage des émissions de dioxyde de carbone ou le remplacement de matière fossile. En tant que réseau de communication ou wood wide web et lieu où la nature innove en permanence, la forêt permet d'introduire le thème de l'industrie connectée et des nouveaux matériaux. L'exposition crée ainsi des parallèles entre le système de communication de la forêt par les racines et par l'air et les technologies les plus avancées de $5 \mathrm{G}$ utilisées par exemple pour les transports ou les sites de production. La signalétique déployée sur les arbres dans le pavillon permet de souligner ces interrelations étroites entre la référence à la forêt et les thématiques abordées dans l'exposition. 
Figure 12. Représentation schématique des rapports entre la forêt et les thématiques de l'exposition

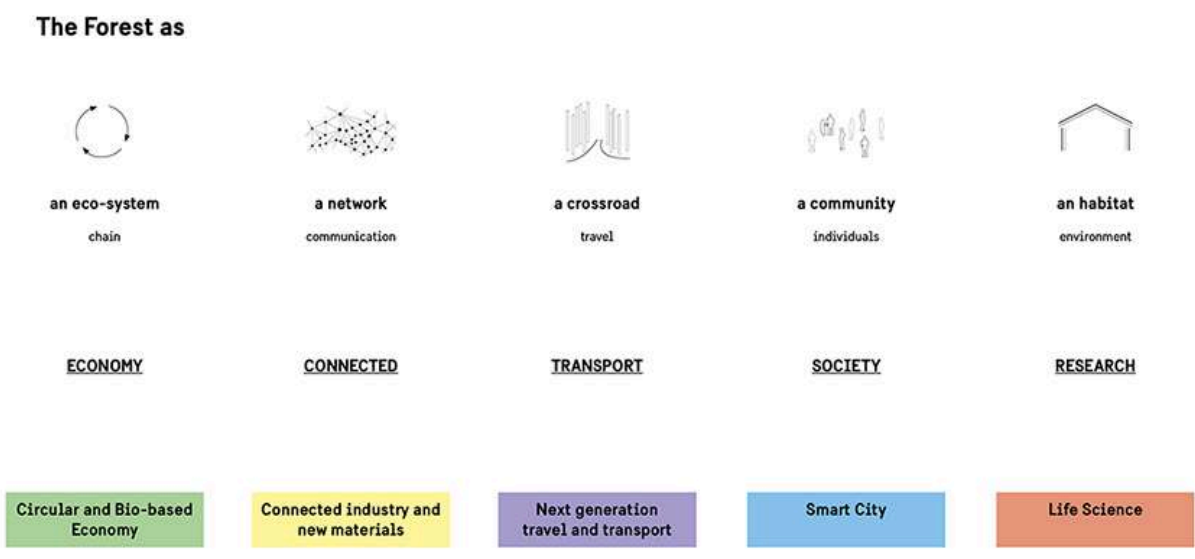

Réalisée en phase d'esquisse, en vue de la construction des contenus de l'exposition.

Source : Studio Adrien Gardère.

En complément des contenus de chaque clairière exposant ces différentes thématiques, des évocations plus directes de l'atmosphère de la forêt sont déployées dans l'exposition. Un paysage sonore diffus est associé ponctuellement à la diffusion de sons de la forêt plus spécifiques, permettant par exemple de plonger le visiteur dans le détail du chant de certains oiseaux, ou du son produit par le vent sur le feuillage.

$\mathrm{Au}$ cœur du parcours de visite, une installation fondée sur un grand écran courbe permet de créer un parallèle entre la forêt fabriquée et abstraite du pavillon et la réelle forêt suédoise. L'installation permet au visiteur, par une interaction simple et intuitive, d'évoluer dans une portion de forêt sauvage suédoise, mais aussi de naviguer entre les saisons, découvrant le même lieu couvert de neige ou bourgeonnant au printemps.

\section{Un écosystème du projet}

Par sa forme à la fois architecturale et scénographique et par les différents niveaux de contenus qu'il véhicule, le pavillon de la Suède construit ainsi différentes strates d'évocations de la forêt. Il permet aux visiteurs de construire leur récit et d'aborder ces différentes épaisseurs narratives au fil de leurs déambulations. Cette découverte commence aux abords du pavillon, où cette forêt architecturale fonctionne comme une image simple et immédiate puis, en franchissant sa lisière, l'exploration se prolonge par une promenade libre entre les arbres et reliefs, donnant accès à différents niveaux d'approfondissement des contenus.

La démarche de collaboration établie en équipe pour "faire pousser» le projet à plusieurs mains a permis de penser le pavillon non pas comme une enveloppe architecturale où s'intègre une scénographie d'exposition, mais plutôt comme une forme d'écosystème opérant comme un tout, où chaque aspect, qu'il soit fonctionnel, formel ou narratif, participe à construire une expérience de visite complète et une lecture de l'espace à plusieurs niveaux. 
cours du processus de conception, le premier niveau intuitif d'utilisation du concept de la forêt est donc rapidement transformé en une véritable matrice de projet opérant à différentes échelles et pour divers aspects. Afin de maintenir cette cohérence tout au long du projet, plusieurs outils et systèmes d'échange ont été mis en place en équipe, qu'il s'agisse du texte manifeste d'introduction, du partage d'images et de projets de référence, de représentations dessinées simples, d'ajustements progressifs du plan, de schémas de construction des contenus. Le pavillon de la Suède s'empare donc d'une image multiforme de la forêt, entre culture et nature, entre art et science, entre forme et récit ${ }^{7}$.

Plus largement, le type de démarche mobilisée dans le cadre de ce projet invite à dépasser les oppositions traditionnelles et le cloisonnement des disciplines encore très présents aujourd'hui dans la conception des lieux d'exposition ou des musées. Si chaque démarche de projet est spécifique et génère des dynamiques de conception toujours uniques et propres au contexte, au programme, à l'équipe réunie, la mise en place de logiques de coconception associant architecture et scénographie, mais aussi programmation et conception des contenus, apparaît comme un enjeu central. Ce sont cependant des processus qui, pour être mis en place, nécessitent des modalités de commande et des méthodes de projet qui gagneraient encore à être explorées. Il s'agit donc de repenser les écosystèmes de projet, non seulement en tant que résultat mais aussi en tant que processus, favorisant le croisement d'approches complémentaires et transdisciplinaires, mobilisant différents métiers et expertises à chacune des échelles et phases du projet.

\section{BIBLIOGRAPHIE}

Chabert, N. et Brun, J. (dir.), Le Rêveur de la forêt, Paris, musée Zadkine/Paris Musées, 2019.

Harrison, R., Forêts. Essai sur l'imaginaire occidental (1992), traduit de l'anglais par F., Naugrette, Paris, Flammarion, coll. « Champs Essais », 1994.

Sompairac, A., Scénographies d'exposition : six perspectives critiques, Genève, Métis Presses, coll. «Vues d'Ensemble Essais », 2016.

Wohlleben, P., La Vie secrète des arbres, Paris, Les Arènes, 2017

\section{NOTES}

1. Les Expositions universelles sont des événements internationaux organisés tous les cinq ans dont les objectifs sont l'éducation du public, la promotion du progrès et la coopération internationale. L'Expo 2020 Dubaï a pour thème « Connecter les esprits, construire le Futur ».

2. C'est au sein du Studio Adrien Gardère que nous menons actuellement un travail de recherche dans le cadre d'une thèse Cifre, associant étroitement pratique du projet et recherche théorique. Cette recherche porte sur les démarches de coconception entre architecture et scénographie lors de la création des musées et lieux d'exposition.

Projets de paysage, 22 | 2020 
3. Parmi les œuvres qui ont inspiré cette recherche, nous pouvons citer The Great Animal Orchestra de Bernie Krause, donnant à entendre toute la subtilité des sons et du chœur que forment les êtres vivants dans leur habitat naturel; Animal Trail de Manabu Miyazaki, capturant d'innombrables scènes de la vie sauvage au Japon par des systèmes photographiques automatiques; les projets Metsä, Intelligent Forest et Bioluminescent Forest, crées par le duo d'artistes Friedrich van Schoor et Tarek Mawad, révélant par des vidéoprojections en forêt des univers surréalistes à la frontière entre technologie et biologie ; le film Il était une forêt, de Luc Jacquet et Francis Hallé, donnant à voir la naissance et la vie d'une forêt tropicale, superposant des images filmées à la poésie de l'animation des dessins botaniques de Francis Hallé.

4. Les anachorètes sont des personnes qui dans l'Antiquité se retiraient dans les déserts notamment d'Égypte et de Syrie. Les ermites qui sont leur équivalent au Moyen Âge menaient leur vie dans les forêts européennes. Ce sont dans les deux cas des personnes vivant retirées du monde, ayant choisi l'ascétisme et la contemplation.

5. Créatures surnaturelles, généralement malfaisantes, appartenant au folklore moyen-oriental ; le désert est l'un de leurs lieux de vie privilégiés.

6. Treillage d'ébénisterie composé de motifs géométriques, placé devant une fenêtre dans les pays orientaux et permettant de voir sans être vu.

7. Cette approche trouve aussi un écho dans la diversité des orientations curatoriales de projets d'exposition récents inspirés par la forêt, tels que l'exposition « Nous les arbres » à la fondation Cartier à Paris, ou l'exposition «Le Rêveur de la forêt " au musée Zadkine à Paris (Chabert et Brun, 2019), témoignant de toute la diversité et de la richesse portées par les formes et l'imaginaire de la forêt.

\section{RÉSUMÉS}

L'article aborde la question de la forêt à travers une approche croisant architecture, paysage, scénographie et programmation. Il est plus particulièrement question de la façon dont une référence culturelle, un symbole et une image - en l'occurrence celle de la forêt - ont servi d'inspiration et d'outils de coconception dans le cadre de la création d'un lieu culturel: le pavillon de la Suède pour l'Exposition universelle de 2020 à Dubaï. Il s'agit ici d'explorer la démarche de conception d'une expérience sensible et sémantique qui exploite à différents niveaux le potentiel esthétique et symbolique de la forêt: une forêt fabriquée, architecturale, dont l'objectif n'est pas d'imiter mais de donner à vivre et ressentir.

The article addresses the subject of the forest through an approach combining architecture, landscape, scenography, and programming. In particular, it focuses on how a cultural reference, a symbol and an image - in this case that of the forest - served as a source of inspiration and as a co-designing tool in the creation of a cultural space : the Swedish pavilion for Expo 2020 in Dubai. The aim is to explore the process of creating a sensory and semantic experience that uses the aesthetic and symbolic potential of the forest on different levels and as an architectural construct which is not intended to imitate the forest but to provide something to be experienced and felt. 
INDEX

Keywords : architectural forest, image, scenography, architecture, co-design

Mots-clés : forêt architecturale, image, scénographie, architecture, coconception

\section{AUTEUR}

\section{MARINA KHÉMIS}

Marina Khémis est designer scénographe et doctorante Cifre au Studio Adrien Gardère à Paris. Ses recherches portent sur les démarches de collaboration articulant scénographie et architecture dans la conception des musées et des lieux d'exposition. Sa thèse est coencadrée par l'ENSA de Versailles (LéaV), ainsi que par l'ENS Paris-Saclay et l'ENSCI Les-Ateliers (CRD). marina_khemis[at]hotmail[dot]fr 